\title{
First measurement of neutrino oscillation parameters using neutrinos and antineutrinos by NOvA
}

M. A. Acero, ${ }^{2}$ P. Adamson, ${ }^{12}$ L. Aliaga, ${ }^{12}$ T. Alion, ${ }^{39}$ V. Allakhverdian, ${ }^{27}$ S. Altakarli, ${ }^{46}$ N. Anfimov, ${ }^{27}$ A. Antoshkin,${ }^{27}$ A. Aurisano, ${ }^{6}$ A. Back,${ }^{24}$ C. Backhouse, ${ }^{44}$ M. Baird,,${ }^{19,39,45}$ N. Balashov, ${ }^{27}$ P. Baldi, ${ }^{25}$ B. A. Bambah, ${ }^{17}$ S. Bashar, ${ }^{43}$ K. Bays, ${ }^{4,21}$ S. Bending, ${ }^{44}$ R. Bernstein, ${ }^{12}$ V. Bhatnagar, ${ }^{32}$ B. Bhuyan, ${ }^{14}$ J. Bian, ${ }^{25,31}$ T. Blackburn, ${ }^{39}$ J. Blair, ${ }^{16}$ A. C. Booth, ${ }^{39}$ P. Bour, ${ }^{9}$ C. Bromberg, ${ }^{29}$ N. Buchanan, ${ }^{8}$ A. Butkevich, ${ }^{22}$ S. Calvez, ${ }^{8}$ M. Campbell, ${ }^{44}$ T. J. Carroll, ${ }^{42}$ E. Catano-Mur, ${ }^{24,47}$ A. Cedeno, ${ }^{46}$ S. Childress, ${ }^{12}$ B. C. Choudhary, ${ }^{11}$ B. Chowdhury, ${ }^{35}$ T. E. Coan, ${ }^{37}$ M. Colo, ${ }^{47}$ J. Cooper, ${ }^{12}$ L. Corwin,${ }^{36}$ L. Cremonesi, ${ }^{44}$ G. S. Davies, ${ }^{19}$ P. F. Derwent, ${ }^{12}$ P. Ding, ${ }^{12}$ Z. Djurcic, ${ }^{1}$ D. Doyle, ${ }^{8}$ E. C. Dukes,${ }^{45}$ H. Duyang, ${ }^{35}$ S. Edayath, ${ }^{7}$ R. Ehrlich, ${ }^{45}$ M. Elkins, ${ }^{24}$ G. J. Feldman, ${ }^{15}$ P. Filip, ${ }^{23}$ W. Flanagan, ${ }^{10}$ M. J. Frank,${ }^{34,45}$ H. R. Gallagher, ${ }^{43}$ R. Gandrajula, ${ }^{29}$ F. Gao, ${ }^{33}$ S. Germani, ${ }^{44}$ A. Giri, ${ }^{18}$ R. A. Gomes, ${ }^{13}$ M. C. Goodman, ${ }^{1}$ V. Grichine, ${ }^{28}$ M. Groh, ${ }^{19}$ R. Group ${ }^{45}$ B. Guo, ${ }^{35}$ A. Habig,${ }^{30}$ F. Hakl,${ }^{20}$ J. Hartnell,${ }^{39}$ R. Hatcher, ${ }^{12}$ A. Hatzikoutelis,${ }^{41}$ K. Heller, ${ }^{31}$ J. Hewes, ${ }^{6}$ A. Himmel, ${ }^{12}$ A. Holin, ${ }^{44}$ B. Howard,${ }^{19}$ J. Huang, ${ }^{42}$ J. Hylen, ${ }^{12}$ F. Jediny, ${ }^{9}$ C. Johnson, ${ }^{8}$ M. Judah, ${ }^{8}$ I. Kakorin, ${ }^{27}$ D. Kalra, ${ }^{32}$ D. M. Kaplan, ${ }^{21}$ R. Keloth, ${ }^{7}$ O. Klimov, ${ }^{27}$ L. W. Koerner, ${ }^{16}$ L. Kolupaeva, ${ }^{27}$ S. Kotelnikov, ${ }^{28}$ I. Kourbanis, ${ }^{12}$ A. Kreymer, ${ }^{12}$ Ch. Kulenberg, ${ }^{27}$ A. Kumar, ${ }^{32}$ C. D. Kuruppu, ${ }^{35}$ V. Kus, ${ }^{9}$ T. Lackey, ${ }^{19}$ K. Lang, ${ }^{42}$ S. Lin, ${ }^{8}$ M. Lokajicek, ${ }^{23}$ J. Lozier, ${ }^{4}$ S. Luchuk, ${ }^{22}$ K. Maan, ${ }^{32}$ S. Magill, ${ }^{1}$ W. A. Mann, ${ }^{43}$ M. L. Marshak, ${ }^{31}$ M. Martinez-Casales, ${ }^{24}$ V. Matveev, ${ }^{22}$ D. P. Méndez, ${ }^{39}$ M. D. Messier $\odot,{ }^{19}$ H. Meyer, ${ }^{46}$ T. Miao, ${ }^{12}$ W. H. Miller, ${ }^{31}$ S. R. Mishra, ${ }^{35}$ A. Mislivec,${ }^{31}$ R. Mohanta, ${ }^{17}$ A. Moren, ${ }^{30}$ L. Mualem, ${ }^{4}$ M. Muether, ${ }^{46}$ S. Mufson, ${ }^{19}$ K. Mulder, ${ }^{44}$ R. Murphy, ${ }^{19}$ J. Musser, ${ }^{19}$ D. Naples,${ }^{33}$ N. Nayak, ${ }^{25}$ J. K. Nelson, ${ }^{47}$ R. Nichol, ${ }^{44}$ G. Nikseresht, ${ }^{21}$ E. Niner, ${ }^{12}$ A. Norman, ${ }^{12}$ T. Nosek, ${ }^{5}$ A. Olshevskiy, ${ }^{27}$ T. Olson, ${ }^{43}$ J. Paley, ${ }^{12}$ R. B. Patterson, ${ }^{4}$ G. Pawloski, ${ }^{31}$ D. Pershey, ${ }^{4}$ O. Petrova, ${ }^{27}$ R. Petti, ${ }^{35}$ D. D. Phan, ${ }^{42}$ R. K. Plunkett, ${ }^{12}$ B. Potukuchi, ${ }^{26}$ C. Principato, ${ }^{45}$ F. Psihas, ${ }^{19,42}$ A. Radovic, ${ }^{47}$ V. Raj ${ }^{4}$ R. A. Rameika, ${ }^{12}$ B. Rebel,${ }^{12,48}$ P. Rojas, ${ }^{8}$ V. Ryabov ${ }^{28}$ O. Samoylov, ${ }^{27}$ M. C. Sanchez, ${ }^{24}$ S. Sánchez Falero, ${ }^{24}$ I. S. Seong, ${ }^{25}$ P. Shanahan, ${ }^{12}$ A. Sheshukov, ${ }^{27}$ P. Singh, ${ }^{11}$ V. Singh, ${ }^{3}$ E. Smith, ${ }^{19}$ J. Smolik, ${ }^{9}$ P. Snopok, ${ }^{21}$ N. Solomey, ${ }^{46}$ E. Song, ${ }^{45}$ A. Sousa, ${ }^{6}$ K. Soustruznik, ${ }^{5}$ M. Strait, ${ }^{31}$ L. Suter, ${ }^{12}$ A. Sutton, ${ }^{45}$ R. L. Talaga, ${ }^{1}$ B. Tapia Oregui, ${ }^{42}$ P. Tas,${ }^{5}$ R. B. Thayyullathil, ${ }^{7}$ J. Thomas, ${ }^{44,48}$ E. Tiras, ${ }^{24}$ D. Torbunov,${ }^{31}$ J. Tripathi, ${ }^{32}$ A. Tsaris, ${ }^{12}$ Y. Torun, ${ }^{21}$ J. Urheim, ${ }^{19}$ P. Vahle, ${ }^{47}$ J. Vasel,${ }^{19}$ L. Vinton, ${ }^{39}$ P. Vokac, ${ }^{9}$ T. Vrba, ${ }^{9}$ M. Wallbank, ${ }^{6}$ B. Wang, ${ }^{37}$ T. K. Warburton, ${ }^{24}$ M. Wetstein, ${ }^{24}$ M. While, ${ }^{36}$ D. Whittington, ${ }^{40,19}$ S. G. Wojcicki, ${ }^{38}$ J. Wolcott, ${ }^{43}$ N. Yadav, ${ }^{14}$ A. Yallappa Dombara, ${ }^{40}$ K. Yonehara, ${ }^{12}$ S. Yu, ${ }^{1,21}$ S. Zadorozhnyy, ${ }^{22}$ J. Zalesak, ${ }^{23}$ B. Zamorano, ${ }^{39}$ and R. Zwaska ${ }^{12}$

(NOvA Collaboration)

\footnotetext{
${ }^{1}$ Argonne National Laboratory, Argonne, Illinois 60439, USA

${ }^{2}$ Universidad del Atlantico, Km. 7 antigua via a Puerto Colombia, Barranquilla, Colombia

${ }^{3}$ Department of Physics, Institute of Science, Banaras Hindu University, Varanasi, 221 005, India

${ }^{4}$ California Institute of Technology, Pasadena, California 91125, USA

${ }^{5}$ Charles University, Faculty of Mathematics and Physics, Institute of Particle and Nuclear Physics, Prague 116 36, Czech Republic

${ }^{6}$ Department of Physics, University of Cincinnati, Cincinnati, Ohio 45221, USA

${ }^{7}$ Department of Physics, Cochin University of Science and Technology, Kochi 682 022, India

${ }^{8}$ Department of Physics, Colorado State University, Fort Collins, Colorado 80523-1875, USA

${ }^{9}$ Czech Technical University in Prague, Brehova 7, 11519 Prague 1, Czech Republic

${ }^{10}$ University of Dallas, 1845 E Northgate Drive, Irving, Texas 75062 USA

${ }^{11}$ Department of Physics and Astrophysics, University of Delhi, Delhi 110007, India

${ }^{12}$ Fermi National Accelerator Laboratory, Batavia, Illinois 60510, USA

${ }^{13}$ Instituto de Física, Universidade Federal de Goiás, Goiânia, Goiás 74690-900, Brazil

${ }^{14}$ Department of Physics, IIT Guwahati, Guwahati 781 039, India

${ }^{15}$ Department of Physics, Harvard University, Cambridge, Massachusetts 02138, USA

${ }^{16}$ Department of Physics, University of Houston, Houston, Texas 77204, USA

${ }^{17}$ School of Physics, University of Hyderabad, Hyderabad 500 046, India

${ }^{18}$ Department of Physics, IIT Hyderabad, Hyderabad 502 205, India

${ }^{19}$ Indiana University, Bloomington, Indiana 47405, USA

${ }^{20}$ Institute of Computer Science, The Czech Academy of Sciences, 18207 Prague, Czech Republic

${ }^{21}$ Department of Physics, Illinois Institute of Technology, Chicago, Illinois 60616, USA

${ }^{22}$ Institute for Nuclear Research of Russia, Academy of Sciences 7a, 60th October Anniversary prospect, Moscow 117312, Russia

${ }^{23}$ Institute of Physics, The Czech Academy of Sciences, 18221 Prague, Czech Republic
} 


\author{
${ }^{24}$ Department of Physics and Astronomy, Iowa State University, Ames, Iowa 50011, USA \\ ${ }^{25}$ Department of Physics and Astronomy, University of California at Irvine, Irvine, California 92697, USA \\ ${ }^{26}$ Department of Physics and Electronics, University of Jammu, Jammu Tawi 180 006, Jammu and Kashmir, India \\ ${ }^{27}$ Joint Institute for Nuclear Research, Dubna, Moscow region 141980, Russia \\ ${ }^{28}$ Nuclear Physics and Astrophysics Division, Lebedev Physical Institute, Leninsky Prospect 53, 119991 Moscow, Russia \\ ${ }^{29}$ Department of Physics and Astronomy, Michigan State University, East Lansing, Michigan 48824, USA \\ ${ }^{30}$ Department of Physics and Astronomy, University of Minnesota Duluth, Duluth, Minnesota 55812, USA \\ ${ }^{31}$ School of Physics and Astronomy, University of Minnesota Twin Cities, Minneapolis, Minnesota 55455, USA \\ ${ }^{32}$ Department of Physics, Panjab University, Chandigarh 160 014, India \\ ${ }^{33}$ Department of Physics, University of Pittsburgh, Pittsburgh, Pennsylvania 15260, USA \\ ${ }^{34}$ Department of Physics, University of South Alabama, Mobile, Alabama 36688, USA \\ ${ }^{35}$ Department of Physics and Astronomy, University of South Carolina, Columbia, South Carolina 29208, USA \\ ${ }^{36}$ South Dakota School of Mines and Technology, Rapid City, South Dakota 57701, USA \\ ${ }^{37}$ Department of Physics, Southern Methodist University, Dallas, Texas 75275, USA \\ ${ }^{38}$ Department of Physics, Stanford University, Stanford, California 94305, USA \\ ${ }^{39}$ Department of Physics and Astronomy, University of Sussex, Falmer, Brighton BN1 9QH, United Kingdom \\ ${ }^{40}$ Department of Physics, Syracuse University, Syracuse, New York 13210, USA \\ ${ }^{41}$ Department of Physics and Astronomy, University of Tennessee, Knoxville, Tennessee 37996, USA \\ ${ }^{42}$ Department of Physics, University of Texas at Austin, Austin, Texas 78712, USA \\ ${ }^{43}$ Department of Physics and Astronomy, Tufts University, Medford, Massachusetts 02155, USA \\ ${ }^{44}$ Physics and Astronomy Dept., University College London, Gower Street, London WC1E 6BT, United Kingdom \\ ${ }^{45}$ Department of Physics, University of Virginia, Charlottesville, Virginia 22904, USA \\ ${ }^{46}$ Department of Mathematics, Statistics, and Physics, Wichita State University, Wichita, Kansas 67206, USA \\ ${ }^{47}$ Department of Physics, College of William \& Mary, Williamsburg, Virginia 23187, USA \\ ${ }^{48}$ Department of Physics, University of Wisconsin-Madison, Madison, Wisconsin 53706, USA
}

(Received 13 June 2019; published 11 October 2019)

\begin{abstract}
The NOvA experiment has seen a $4.4 \sigma$ signal of $\bar{\nu}_{e}$ appearance in a $2 \mathrm{GeV} \bar{\nu}_{\mu}$ beam at a distance of $810 \mathrm{~km}$. Using $12.33 \times 10^{20}$ protons on target delivered to the Fermilab NuMI neutrino beamline, the experiment recorded $27 \bar{\nu}_{\mu} \rightarrow \bar{\nu}_{e}$ candidates with a background of 10.3 and $102 \bar{\nu}_{\mu} \rightarrow \bar{\nu}_{\mu}$ candidates. This new antineutrino data are combined with neutrino data to measure the parameters $\left|\Delta m_{32}^{2}\right|=2.48_{-0.06}^{+0.11} \times$ $10^{-3} \mathrm{eV}^{2} / c^{4}$ and $\sin ^{2} \theta_{23}$ in the ranges from (0.53-0.60) and (0.45-0.48) in the normal neutrino mass hierarchy. The data exclude most values near $\delta_{C P}=\pi / 2$ for the inverted mass hierarchy by more than $3 \sigma$ and favor the normal neutrino mass hierarchy by $1.9 \sigma$ and $\theta_{23}$ values in the upper octant by $1.6 \sigma$.
\end{abstract}

DOI: 10.1103/PhysRevLett.123.151803

The observations of neutrino oscillations by many experiments [1-9] are well described by the mixing of three neutrino mass eigenstates $\nu_{1}, \nu_{2}$, and $\nu_{3}$ with the flavor eigenstates $\nu_{e}, \nu_{\mu}$, and $\nu_{\tau}$. The mixing is parametrized by a unitary matrix which depends on three angles and a phase, $\delta_{C P}$, that may break charge-parity $(C P)$ symmetry. The oscillation frequencies are proportional to the neutrino mass splittings, $\Delta m_{21}^{2} \equiv m_{2}^{2}-m_{1}^{2} \simeq 7.5 \times 10^{-5} \mathrm{eV}^{2} / c^{4}$ and $\left|\Delta m_{32}^{2}\right| \simeq 2.5 \times 10^{-3} \mathrm{eV}^{2} / \mathrm{c}^{4}$, and the angles are known to be large: $\theta_{12} \simeq 34^{\circ}, \theta_{13} \simeq 8^{\circ}, \theta_{23} \simeq 45^{\circ}$ [10]; $\delta_{C P}$, however, is largely unknown.

Within this framework, several questions remain unanswered. The angle $\theta_{23}$ produces nearly maximal mixing but

Published by the American Physical Society under the terms of the Creative Commons Attribution 4.0 International license. Further distribution of this work must maintain attribution to the author(s) and the published article's title, journal citation, and DOI. Funded by SCOAP ${ }^{3}$. has large uncertainties. If maximal, it would introduce an unexplained $\mu-\tau$ symmetry; should it differ from $45^{\circ}$, its octant would determine whether $\nu_{\tau}$ or $\nu_{\mu}$ couples more strongly to $\nu_{3}$. Furthermore, while it is known that the two independent mass splittings differ by a factor of 30 , the sign of the larger splitting is unknown. The $\nu_{1}$ and $\nu_{2}$ states that contribute most to the $\nu_{e}$ state could be lighter ["normal hierarchy" (NH)] or heavier ["inverted hierarchy" (IH)] than the $\nu_{3}$ state. This question has important implications for models of neutrino mass [11-15] and for the study of the Dirac vs Majorana nature of the neutrino [16,17]. Additionally, neutrino mixing may be a source of $C P$ violation if $\sin \delta_{C P}$ is nonzero.

These questions can be addressed by the measurement of $\nu_{\mu} \rightarrow \nu_{\mu}, \bar{\nu}_{\mu} \rightarrow \bar{\nu}_{\mu}, \nu_{\mu} \rightarrow \nu_{e}$, and $\bar{\nu}_{\mu} \rightarrow \bar{\nu}_{e}$ oscillations in matter over baselines $L$ of order (100-1000) km, with neutrino energies $E[\mathrm{GeV}] \simeq L[\mathrm{~km}] \times\left|\Delta m_{32}^{2}\left[\mathrm{eV}^{2} / c^{4}\right]\right|$. Several long-baseline experiments have reported observations of $\nu_{\mu} \rightarrow \nu_{\mu}$ [18-21], $\nu_{\mu} \rightarrow \nu_{e}$ [19-21], and $\bar{\nu}_{\mu} \rightarrow \bar{\nu}_{\mu}$ $[19,20]$, but a statistically significant observation of 
$\bar{\nu}_{\mu} \rightarrow \bar{\nu}_{e}$ has not previously been made. This report combines the first antineutrino measurements by NOvA with the neutrino data reported in Ref. [21] in a reoptimized analysis yielding a new determination of the oscillation parameters $\left|\Delta m_{32}^{2}\right|, \sin ^{2} \theta_{23}, \delta_{C P}$, and the neutrino mass hierarchy.

The NOvA experiment measures oscillations by comparing the energy spectra of neutrino interactions in two detectors placed in the Fermilab NuMI beam [22] at distances of $1 \mathrm{~km}$ [near detector (ND)] and $810 \mathrm{~km}$ [far detector (FD)] from the production target. The 14 kton FD and 290 ton ND are sampling calorimeters constructed from PVC and liquid scintillator [23,24]. The ND is located $100 \mathrm{~m}$ underground. The FD operates on the surface with modest shielding resulting in $130 \mathrm{kHz}$ of cosmic-ray activity. The detectors are located $14.6 \mathrm{mrad}$ off the beam axis where the neutrino energy spectrum peaks at $2 \mathrm{GeV}$. Magnetic focusing horns in the beamline charge-select neutrino parents giving $96 \%(83 \%)$ pure $\nu_{\mu}\left(\bar{\nu}_{\mu}\right)$ event samples between 1 and $5 \mathrm{GeV}$. Most contamination is "wrong sign" ( $\bar{\nu}$ in the $\nu$ beam, or vice versa) with $<1 \% \nu_{e}+\bar{\nu}_{e}$.

This Letter reports data from an antineutrino run spanning from June 29, 2016, to February 26, 2019, with an exposure of $12.33 \times 10^{20}$ protons on target (POT) delivered during $317.0 \mathrm{~s}$ of beam-on time, combined with the previously reported [21] neutrino beam exposure of $8.85 \times 10^{20}$ POT and $438.2 \mathrm{~s}$. During these periods, the proton source achieved a peak hourly averaged power of $742 \mathrm{~kW}$.

The flux of neutrinos delivered to the detectors is calculated using a simulation of the production and transport of particles through the beamline components [22,25] reweighted to incorporate external measurements [26-45]. Neutrino interactions in the detector are simulated using GENIE [46] tuned to improve agreement with external measurements and ND data, reducing uncertainties in the extrapolation of measurements in the ND to the FD. As in Ref. [21], we set $M_{A}$ in the quasielastic dipole form factor to $1.04 \mathrm{GeV} / c^{2}$ [47] and use corrections to the chargedcurrent (CC) quasielastic cross section derived from the random phase approximation $[48,49]$. In this analysis, we also apply this effect to baryon resonances as a placeholder for the unknown nuclear effect that suppresses rates at a low four-momentum transfer in our and other measurements [50-53]. Additionally, we increase the rate of deep-inelastic scattering with hadronic mass $W>1.7 \mathrm{GeV} / c^{2}$ by $10 \%$ to match our observed counts of short track-length $\nu_{\mu}$ CC events. We model multinucleon ejection interactions following Ref. [54] and adjust the rates in bins of energy transfer, $q_{0}$, and three-momentum transfer, $|\vec{q}|$, for $\nu_{\mu}$ and $\bar{\nu}_{\mu}$ separately to maximize agreement in the ND. The calculation of the $\nu_{e}$ and $\bar{\nu}_{e}$ rates uses these same models.

The energy depositions of final-state particles are simulated with GEANT4 [25] and input to a custom simulation of the detector response [55]. The absolute energy scale of the detectors is calibrated to within $\pm 5 \%$ using the minimum ionizing portion of cosmic-ray muon tracks that stop in the detectors.

Cells with activity above threshold (hits) are grouped based on their proximity in space and time to produce candidate neutrino events. Events are assigned a vertex, and clusters are formed from hits likely to be associated with particles produced there [56]. These clusters are categorized as electromagnetic or hadronic in origin using a convolutional neural network (CNN) [57]. Hits forming tracks are identified as muons by combining information on the track length, $d E / d x$, vertex activity, and scattering into a single particle identification (PID) score [58]. The same reconstruction algorithms are applied to events from data and simulation in both detectors.

The $\nu_{\mu}$ and $\bar{\nu}_{\mu}$ candidates are required to have a vertex inside the fiducial volume and no evidence of particles exiting the detector. Following Ref. [21], the $\nu_{e}$ and $\bar{\nu}_{e}$ candidates are divided into a "core" sample which satisfies these containment requirements, and a "peripheral" sample which loosens these requirements for the most signal-like event topologies. A second CNN [59] serves as the primary PID, classifying events as $\nu_{e} \mathrm{CC}, \nu_{\mu} \mathrm{CC}, \nu_{\tau} \mathrm{CC}$, neutral current (NC), or cosmic ray. The network is trained on simulated neutrino and antineutrino beam conditions and cosmic-ray data. It has an improved architecture and higher rate of cosmic ray rejection over the previous network [21]. Events identified as $\nu_{\mu} \mathrm{CC}$ are required to contain at least one track classified as a muon.

Several requirements target cosmic-ray backgrounds. For the $\nu_{\mu}$ CC sample, a boosted decision tree (BDT) algorithm based on vertex position and muonlike track properties is used. Events in the core $\nu_{e}$ sample not aligned with the beam direction and that are near the top of the detector are rejected. Events are removed whose topology is consistent with detached bremsstrahlung showers from cosmic tracks and with photons entering from the detector's north side where there is less shielding. Cosmic-ray backgrounds in the $\nu_{e}$ peripheral sample are removed with a BDT based on position and direction information.

The selection of $\nu_{\mu}$ and $\bar{\nu}_{\mu}$ CC events is $31.2 \%$ (33.9\%) efficient for true interactions in the fiducial volume, resulting in $98.6 \%$ (98.8\%) pure samples at the FD during neutrino (antineutrino) beam operation. Both $\nu_{\mu}$ and $\bar{\nu}_{\mu}$ are counted as a signal for the disappearance measurements. Selections against exiting particle tracks are the largest source of inefficiency. The efficiency for selecting signal $\nu_{e}$ $\mathrm{CC}\left(\bar{\nu}_{e} \mathrm{CC}\right)$ events is $62 \%(67 \%)$. Purities for the signal $\nu_{e}$ $\left(\bar{\nu}_{e}\right)$ samples fall in the range $57-78 \%(55-77 \%)$ depending on the impact of oscillations on the signal and wrong-sign background levels. These efficiencies and purities differ from those quoted in Ref. [21] due to a reoptimization of the selection algorithms [60]. The wrong-sign component of the selected $\nu_{\mu}$ sample in the ND is calculated to be 

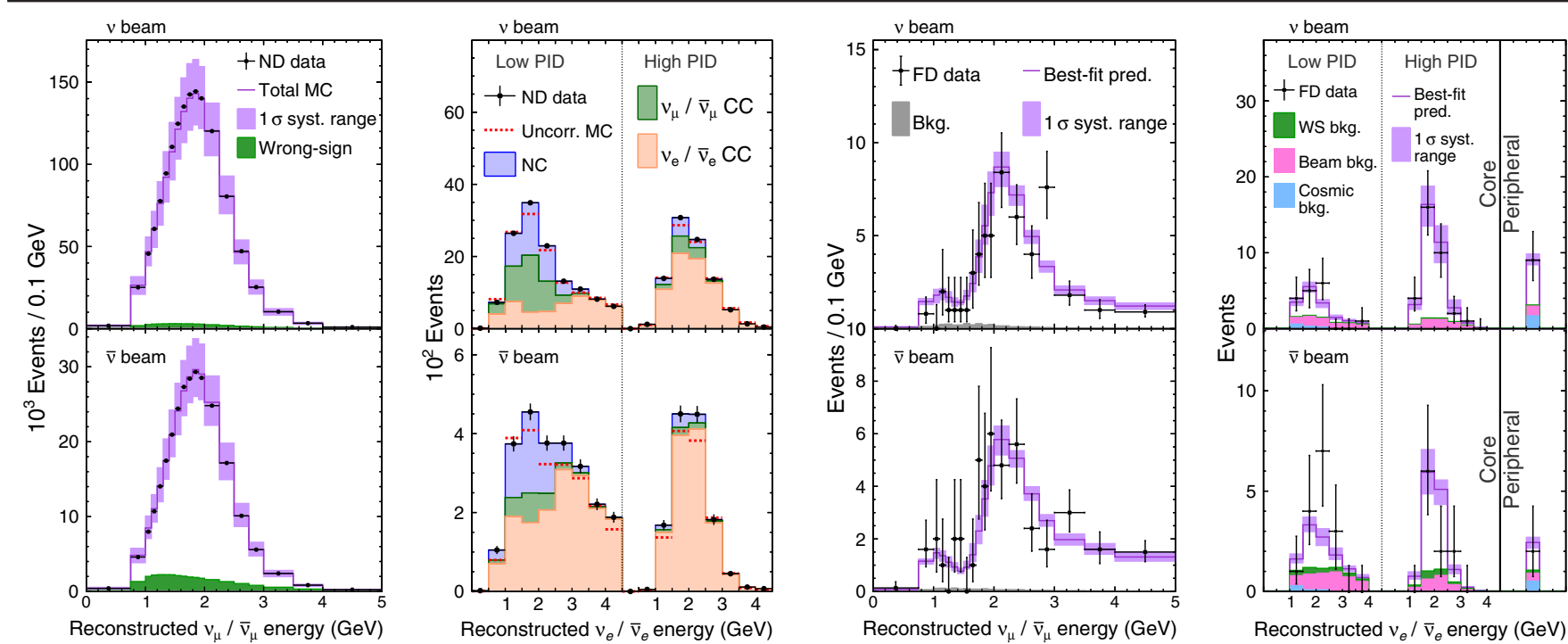

FIG. 1. From left to right, the reconstructed neutrino energy spectra for the ND $\nu_{\mu} \mathrm{CC}, \mathrm{ND} \nu_{e} \mathrm{CC}, \mathrm{FD} \nu_{\mu} \mathrm{CC}, \mathrm{FD} \nu_{e} \mathrm{CC}[61]$ with neutrino data shown across the top and antineutrino data across the bottom. For the ND $\nu_{\mu}$ CC spectra, backgrounds, aside from wrongsign candidates, are negligible and not shown. The $\nu_{e}$ CC spectra are split into a low and high purity sample, and the FD spectra shows counts in the "peripheral" sample. The dashed lines in the ND $\nu_{e}$ spectra show the totals before data-driven corrections.

$2.8 \pm 0.3 \%$ and $10.6 \pm 1.1 \%$ for the neutrino and antineutrino beams. These fractions are consistent with a datadriven estimate based on the rate of $\nu_{\mu} \mathrm{CC}$ and $\mathrm{NC}$ interactions with associated detector activity indicative of neutron capture.

The incident neutrino energy is reconstructed from the measured energies of the final-state lepton and recoil hadronic system. The lepton energy is estimated from track length for muon candidates and from calorimetric energy for electron candidates. The hadronic energy is estimated from the sum of the calibrated hits not associated with the primary lepton. The neutrino energy resolution at the FD is $9.1 \%(8.1 \%)$ for $\nu_{\mu} \mathrm{CC}\left(\bar{\nu}_{\mu} \mathrm{CC}\right)$ events and $10.7 \%$ $(8.8 \%)$ for $\nu_{e} \mathrm{CC}\left(\bar{\nu}_{e} \mathrm{CC}\right)$ events. We analyze the $\nu_{\mu}$ and $\bar{\nu}_{\mu}$ events in quartiles of hadronic energy fraction as events with less hadronic energy have the best energy resolution and lowest backgrounds [21].

The energy spectra of the selected $\nu_{\mu} \mathrm{CC}$ and $\nu_{e} \mathrm{CC}$ interactions in the ND during neutrino and antineutrino beam operations are shown in Fig. 1. The selected ND $\nu_{e}$ sample consists entirely of background sources for the $\nu_{e}$ appearance measurement, predominantly the intrinsic beam $\nu_{e}$ component, along with misidentified $\nu_{\mu} \mathrm{CC}$ and $\mathrm{NC}$ interactions. We analyze the $\nu_{e}$ candidate energy spectra in two bins of $\nu_{e}$ PID ("low" and "high") to isolate a highly pure sample of $\nu_{\mu} \rightarrow \nu_{e}$ and $\bar{\nu}_{\mu} \rightarrow \bar{\nu}_{e}$ at the FD. In the ND, the high-PID sample is dominated by intrinsic beam $\nu_{e}$. A third bin containing the "peripheral" events is added for the FD.

The $\nu_{\mu}$ and $\nu_{e}$ signal spectra at the FD are predicted for the neutrino and antineutrino beams separately using the observed spectra of $\nu_{\mu}$ candidate events in the ND. The true neutrino energy spectrum at the ND is estimated using the measured event rates in bins of reconstructed energy and the energy distributions of simulated events found to populate those bins. This true spectrum is corrected for differences in flux and acceptance between the ND and FD, as well as differences in the $\nu_{\mu}$ and $\nu_{e}$ cross sections;

TABLE I. Systematic uncertainties on the total predicted numbers of signal and beam-related background events at the best fit point (see Table IV) in the $\nu_{e}$ selected samples in the neutrino and antineutrino datasets.

\begin{tabular}{lcccr}
\hline \hline & $\nu_{e}$ signal & $\nu_{e}$ bkg. & $\bar{\nu}_{e}$ signal & $\bar{\nu}_{e}$ bkg. \\
\cline { 2 - 5 } Source & $(\%)$ & $(\%)$ & $(\%)$ & $(\%)$ \\
\hline Cross sections & $+4.7 /-5.8$ & $+3.6 /-3.4$ & $+3.2 /-4.2$ & $+3.0 /-2.9$ \\
Detector model & $+3.7 /-3.9$ & $+1.3 /-0.8$ & $+0.6 /-0.6$ & $+3.7 /-2.6$ \\
ND and FD differences & $+3.4 /-3.4$ & $+2.6 /-2.9$ & $+4.3 /-4.3$ & $+2.8 /-2.8$ \\
Calibration & $+2.1 /-3.2$ & $+3.5 /-3.9$ & $+1.5 /-1.7$ & $+2.9 /-0.5$ \\
Others & $+1.6 /-1.6$ & $+1.5 /-1.5$ & $+1.4 /-1.2$ & $+1.0 /-1.0$ \\
\hline Total & $+7.4 /-8.5$ & $+5.6 /-6.2$ & $+5.8 /-6.4$ & $+6.3 /-4.9$ \\
\hline \hline
\end{tabular}


TABLE II. Systematic uncertainties on the oscillation parameters $\sin ^{2} \theta_{23}, \Delta m_{32}^{2}$, and $\delta_{C P}$, evaluated at the best fit point (see Table IV).

\begin{tabular}{lccc}
\hline \hline & $\sin ^{2} \theta_{23}$ & $\left|\Delta m_{32}^{2}\right|$ & $\delta_{C P}$ \\
\cline { 2 - 4 } Source & $\left(10^{-3}\right)$ & $\left(10^{-5} \mathrm{eV}^{2} / c^{4}\right)$ & $(\pi)$ \\
\hline Calibration & $+5.4 /-9.2$ & $+2.2 /-2.6$ & $+0.03 /-0.03$ \\
Neutron model & $+6.0 /-13.0$ & $+0.5 /-1.3$ & $+0.01 /-0.00$ \\
Cross sections & $+4.1 /-7.7$ & $+1.0 /-1.1$ & $+0.06 /-0.07$ \\
$E_{\mu}$ scale & $+2.3 /-3.0$ & $+1.0 /-1.1$ & $+0.00 /-0.00$ \\
Detector model & $+1.9 /-3.2$ & $+0.4 /-0.5$ & $+0.05 /-0.05$ \\
Normalizations & $+1.3 /-2.7$ & $+0.1 /-0.2$ & $+0.02 /-0.03$ \\
ND and & $+1.0 /-4.0$ & $+0.2 /-0.2$ & $+0.06 /-0.07$ \\
$\quad$ FD diffs. & & & \\
Beam flux & $+0.4 /-0.8$ & $+0.1 /-0.1$ & $+0.00 /-0.00$ \\
\hline Total systematic & $+9.7 /-20$ & $+2.6 /-3.2$ & $+0.11 /-0.12$ \\
\hline \hline
\end{tabular}

oscillations are applied to yield predictions for the true $\nu_{\mu}$ and $\nu_{e}$ spectra at the FD. These spectra are then transformed into reconstructed energy using the underlying energy distributions from simulated neutrino interactions in the FD.

The predicted background spectra at the FD are also primarily data driven. Data collected out of time with the NuMI beam provide a measurement of the rate of cosmicray backgrounds in the $\nu_{\mu}$ and $\nu_{e}$ samples. Neutrino backgrounds calculated to populate the $\mathrm{FD} \nu_{e}$ spectra are corrected based on the reconstructed $\nu_{e}$ candidates at the ND. The procedure from Ref. [21] is followed to determine corrections for each background component in the neutrino-mode beam, while for the antineutrino-mode beam a single scale factor is used. The remaining backgrounds, which include any misidentified neutrino events

TABLE III. Event counts at the FD, both observed and predicted at the best fit point (see Table IV).

\begin{tabular}{lccccc}
\hline \hline & \multicolumn{2}{c}{ Neutrino beam } & & \multicolumn{2}{c}{ Antineutrino beam } \\
\cline { 2 - 3 } \cline { 6 - 6 } \cline { 5 - 6 } & $\nu_{\mu} \mathrm{CC}$ & $\nu_{e} \mathrm{CC}$ & & $\bar{\nu}_{\mu} \mathrm{CC}$ & $\bar{\nu}_{e} \mathrm{CC}$ \\
\hline$\nu_{\mu} \rightarrow \nu_{\mu}$ & 112.5 & 0.7 & & 24.0 & 0.1 \\
$\bar{\nu}_{\mu} \rightarrow \bar{\nu}_{\mu}$ & 7.2 & 0.0 & & 70.0 & 0.1 \\
$\nu_{\mu} \rightarrow \nu_{e}$ & 0.1 & 44.3 & & 0.0 & 2.2 \\
$\bar{\nu}_{\mu} \rightarrow \bar{\nu}_{e}$ & 0.0 & 0.6 & & 0.0 & 16.6 \\
Beam $\nu_{e}+\bar{\nu}_{e}$ & 0.0 & 7.0 & & 0.0 & 5.3 \\
NC & 1.3 & 3.1 & & 0.8 & 1.2 \\
Cosmic & 2.1 & 3.3 & & 0.8 & 1.1 \\
Others & 0.7 & 0.4 & & 0.6 & 0.3 \\
\hline Signal & $119.7_{-11.8}^{+10.2}$ & $44.3_{-4.0}^{+3.5}$ & & $93.9_{-8.2}^{+8.1}$ & $16.6_{-1.0}^{+0.9}$ \\
Background & $4.2_{-0.6}^{+0.5}$ & $15.0_{-0.9}^{+0.8}$ & & $2.2_{-0.4}^{+0.4}$ & $10.3_{-0.5}^{+0.6}$ \\
\hline Best fit & 123.9 & 59.3 & & 96.2 & 26.8 \\
Observed & 113 & 58 & & 102 & 27 \\
\hline \hline
\end{tabular}

in the $\nu_{\mu}$ samples and misidentified $\nu_{\tau}$ interactions in the $\nu_{e}$ samples, make up less than $2 \%$ of the FD candidates and are taken directly from simulation.

To evaluate the impact of systematic uncertainties we recompute the extrapolation from the ND to the FD varying the parameters used to model the neutrino fluxes, neutrino cross sections, and detector response. The procedure accounts for changes in the composition of the $\nu_{e}$ background, and for impacts on the transformation to and from true and reconstructed energies due to variations in the model parameters. We parametrize each systematic variation and compute its effect in each analysis bin. These parameters are included in the oscillation fit, constrained within their estimated uncertainties by penalty terms in the likelihood function.

The oscillation parameters that best fit the FD data are determined through minimization of a Poisson negative $\log$-likelihood, $-2 \ln \mathcal{L}$, varying three unconstrained parameters, $\Delta m_{32}^{2}, \sin ^{2} \theta_{23}$, and $\delta_{C P}$, as well as 53 constrained parameters covering the other oscillation parameters and the sources of systematic uncertainty summarized in Tables I and II. The two-detector design and extrapolation procedure significantly reduce the effect of the $\simeq 10-20 \%$ a priori uncertainties on the beam flux and cross sections. The principal remaining uncertainties are neutrino cross sections, the energy scale calibration, the detector response to neutrons, and differences between the ND and FD that cannot be corrected by extrapolation.

The selection criteria and techniques used in the analysis were developed on simulated data prior to inspection of the FD data distributions. Figure 1 shows the energy spectra of the $\nu_{\mu} \mathrm{CC}, \bar{\nu}_{\mu} \mathrm{CC}, \nu_{e} \mathrm{CC}$, and $\bar{\nu}_{e} \mathrm{CC}$ candidates recorded at the FD overlaid on their oscillated best-fit expectations. Table III summarizes the total event counts and estimated compositions of the selected samples. We recorded $102 \bar{\nu}_{\mu}$

TABLE IV. Summary of oscillation parameters. The top three are inputs to this analysis [10], while the rest are the best fits for different choices of the mass hierarchy $(\mathrm{NH}, \mathrm{IH})$ and $\theta_{23}$ octant (UO, LO), along with the significance (in units of $\sigma$ ) at which those combinations are disfavored. In addition to the region indicated, for $\mathrm{NH}, \mathrm{LO}$ a small range of $\sin ^{2} \theta_{23} 0.45-0.48$ is allowed at $1 \sigma[61]$.

\begin{tabular}{|c|c|c|c|c|}
\hline$\Delta m_{21}^{2} /\left(10^{-5} \mathrm{eV}^{2} / c^{4}\right)$ & \multicolumn{4}{|c|}{$7.53 \pm 0.18$} \\
\hline $\sin ^{2} \theta_{12}$ & \multicolumn{4}{|c|}{$0.307_{-0.012}^{+0.013}$} \\
\hline $\sin ^{2} \theta_{13}$ & \multicolumn{4}{|c|}{$0.0210 \pm 0.0011$} \\
\hline & NH, UO & NH, LO & IH, UO & $\mathrm{IH}, \mathrm{LO}$ \\
\hline$\Delta m_{32}^{2} /\left(10^{-3} \mathrm{eV}^{2} / c^{4}\right)$ & $+2.48_{-0.06}^{+0.11}$ & +2.47 & -2.54 & -2.53 \\
\hline $\sin ^{2} \theta_{23}$ & $0.56_{-0.03}^{+0.04}$ & 0.48 & 0.56 & 0.47 \\
\hline \multirow[t]{2}{*}{$\delta_{C P} / \pi$} & $0.0_{-0.4}^{+1.3}$ & 1.9 & 1.5 & 1.4 \\
\hline & - & $+1.6 \sigma$ & $+1.8 \sigma$ & $+2.0 \sigma$ \\
\hline
\end{tabular}




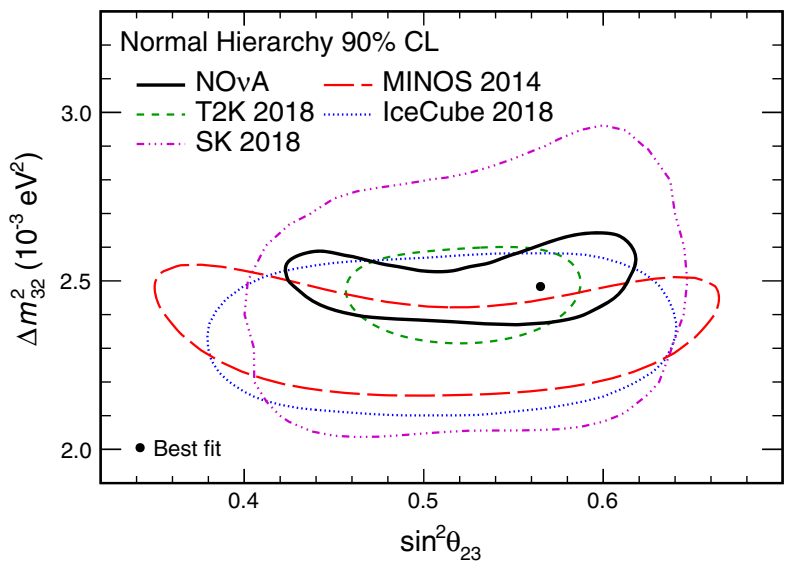

FIG. 2. The $90 \%$ confidence level region for $\Delta m_{32}^{2}$ and $\sin ^{2} \theta_{23}$, with best-fit point shown as a black marker [61], overlaid on contours from other experiments $[19,20,64,65]$.

candidate events at the FD, reflecting a significant suppression from the unoscillated expectation of 476. We find $27 \bar{\nu}_{\mu} \rightarrow \bar{\nu}_{e}$ candidate events with an estimated background of $10.3_{-0.5}^{+0.6}$, a $4.4 \sigma$ excess over the predicted background.

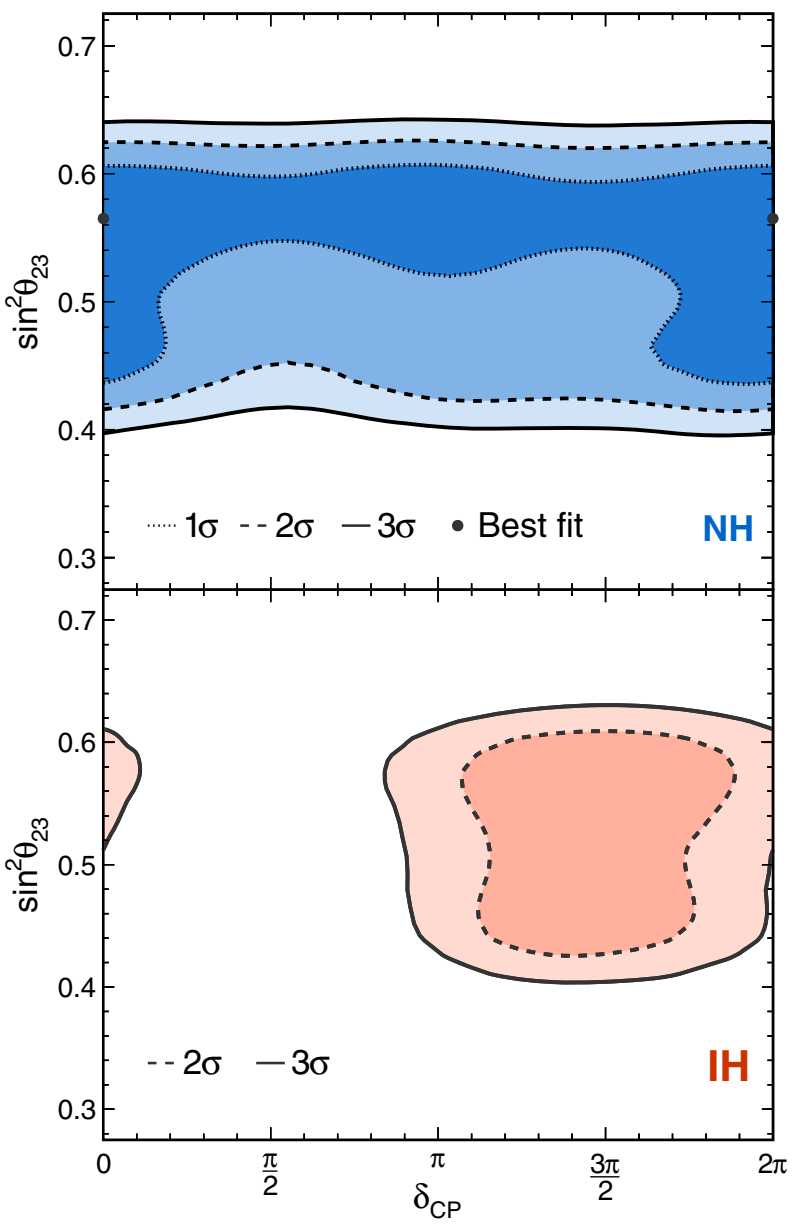

FIG. 3. The $1 \sigma, 2 \sigma$, and $3 \sigma$ contours in $\sin ^{2} \theta_{23}$ vs $\delta_{C P}$ in the normal hierarchy (NH, top panel) and inverted hierarchy ( $\mathrm{IH}$, bottom panel) [61]. The best-fit point is shown by a black marker.
This observation is the first evidence of $\bar{\nu}_{e}$ appearance in a $\bar{\nu}_{\mu}$ beam over a long baseline. These new antineutrino data are analyzed together with $113 \nu_{\mu}$ and $58 \nu_{\mu} \rightarrow \nu_{e}$ candidates from the previous data set.

Table IV shows the overall best-fit parameters and the best fits for each choice of $\theta_{23}$ octant and hierarchy. The best-fit point is found for the normal hierarchy with $\theta_{23}$ in the upper octant where $-2 \ln \mathcal{L}=157.1$ for 175 degrees of freedom (goodness of fit $p=0.91$ from simulated experiments). The measured values of $\theta_{23}$ and $\Delta m_{32}^{2}$ are consistent with the previous NOvA measurement [21] that used only neutrino data, and are consistent with maximal mixing within $1.2 \sigma$.

Confidence intervals for the oscillation parameters are determined using the unified approach $[62,63]$. Figure 2 compares the $90 \%$ confidence level contours in $\Delta m_{32}^{2}$ and $\sin ^{2} \theta_{23}$ with those of other experiments $[19,20,64,65]$. Figure 3 shows the allowed regions in $\sin ^{2} \theta_{23}$ and $\delta_{C P}$. These results exclude $\delta_{C P}$ values in the inverted mass hierarchy from -0.04 to $0.97 \pi$ in the lower $\theta_{23}$ octant and 0.04 to $0.91 \pi$ in the upper octant by more than $3 \sigma$. The data prefer the normal hierarchy with a significance of $1.9 \sigma$ ( $p=0.057, \mathrm{CL}_{s}=0.091$ [66]) and the upper $\theta_{23}$ octant with a significance of $1.6 \sigma(p=0.11)$ [67].

We are grateful to Stephen Parke (FNAL) for useful discussions. This document was prepared by the NOvA collaboration using the resources of the Fermi National Accelerator Laboratory (Fermilab), a U.S. Department of Energy, Office of Science, HEP User Facility. Fermilab is managed by Fermi Research Alliance, LLC (FRA), acting under Contract No. DE-AC02-07CH11359. This work was supported by the U.S. Department of Energy; the U.S. National Science Foundation; the Department of Science and Technology, India; the European Research Council; the MSMT CR, GA UK, Czech Republic; the RAS, RFBR, RMES, RSF, and BASIS Foundation, Russia; CNPq and FAPEG, Brazil; STFC, and the Royal Society, United Kingdom; and the state and University of Minnesota. This work used resources of the National Energy Research Scientific Computing Center (NERSC), a U.S. Department of Energy Office of Science User Facility operated under Contract No. DE-AC02-05CH11231. We are grateful for the contributions of the staffs of the University of Minnesota at the Ash River Laboratory and of Fermilab.

[1] Y. Fukuda et al. (Super-Kamiokande Collaboration), Phys. Rev. Lett. 81, 1562 (1998).

[2] S. Fukuda et al. (Super-Kamiokande Collaboration), Phys. Lett. B 539, 179 (2002).

[3] Q. R. Ahmad et al. (SNO Collaboration), Phys. Rev. Lett. 89, 011301 (2002).

[4] K. Eguchi et al. (KamLAND Collaboration), Phys. Rev. Lett. 90, 021802 (2003). 
[5] D. G. Michael et al. (MINOS Collaboration), Phys. Rev. Lett. 97, 191801 (2006).

[6] K. Abe et al. (T2K Collaboration), Phys. Rev. Lett. 107, 041801 (2011).

[7] Y. Abe et al. (Double Chooz Collaboration), Phys. Rev. Lett. 108, 131801 (2012).

[8] F. P. An et al. (Daya Bay Collaboration), Phys. Rev. Lett. 108, 171803 (2012).

[9] J. K. Ahn et al. (RENO Collaboration), Phys. Rev. Lett. 108, 191802 (2012).

[10] C. Patrignani et al. (Particle Data Group), Chin. Phys. C 40, 100001 (2016), and 2017 update.

[11] R. N. Mohapatra and A. Y. Smirnov, Annu. Rev. Nucl. Part. Sci. 56, 569 (2006).

[12] H. Nunokawa, S. J. Parke, and J. W. F. Valle, Prog. Part. Nucl. Phys. 60, 338 (2008).

[13] G. Altarelli and F. Feruglio, Rev. Mod. Phys. 82, 2701 (2010).

[14] S. F. King, J. Phys. G 42, 123001 (2015).

[15] S. T. Petcov, Eur. Phys. J. C 78, 709 (2018).

[16] S. Pascoli and S. T. Petcov, Phys. Lett. B 544, 239 (2002).

[17] J. N. Bahcall, H. Murayama, and C. Pena-Garay, Phys. Rev. D 70, 033012 (2004).

[18] M. H. Ahn et al. (K2K Collaboration), Phys. Rev. D 74, 072003 (2006).

[19] P. Adamson et al. (MINOS Collaboration), Phys. Rev. Lett. 112, 191801 (2014).

[20] K. Abe et al. (T2K Collaboration), Phys. Rev. Lett. 121, 171802 (2018).

[21] M. A. Acero et al. (NOvA Collaboration), Phys. Rev. D 98, 032012 (2018).

[22] P. Adamson et al., Nucl. Instrum. Methods Phys. Res., Sect. A 806, 279 (2016).

[23] D.S. Ayres et al. (NOvA Collaboration), The NOvA Technical Design Report, United States, 2007.

[24] S. Mufson et al., Nucl. Instrum. Methods Phys. Res., Sect. A 799, 1 (2015).

[25] S. Agostinelli et al. (GEANT4 Collaboration), Nucl. Instrum. Methods Phys. Res., Sect. A 506, 250 (2003).

[26] L. Aliaga et al. (MINERvA Collaboration), Phys. Rev. D 94, 092005 (2016); 95, 039903(A) (2017).

[27] J. M. Paley et al. (MIPP Collaboration), Phys. Rev. D 90, 032001 (2014).

[28] C. Alt et al. (NA49 Collaboration), Eur. Phys. J. C 49, 897 (2007).

[29] N. Abgrall et al. (NA61/SHINE Collaboration), Phys. Rev. C 84, 034604 (2011).

[30] D. S. Barton et al., Phys. Rev. D 27, 2580 (1983).

[31] S. M. Seun, Measurement of $\pi-K$ ratios from the NuMI target, Ph.D. thesis, Harvard University, 2007.

[32] G. M. Tinti, Sterile neutrino oscillations in MINOS and hadron production in $\mathrm{pC}$ collisions, Ph.D. thesis, Oxford University, 2010.

[33] A. V. Lebedev, Ratio of pion kaon production in proton carbon interactions, Ph.D. thesis, Harvard University, 2007.

[34] B. Baatar et al. (NA49 Collaboration), Eur. Phys. J. C 73, 2364 (2013).

[35] P. Skubic et al., Phys. Rev. D 18, 3115 (1978).
[36] S. P. Denisov, S. V. Donskov, Yu. P. Gorin, R. N. Krasnokutsky, A. I. Petrukhin, Yu. D. Prokoshkin, and D. A. Stoyanova, Nucl. Phys. B61, 62 (1973).

[37] A. S. Carroll et al., Phys. Lett. 80B, 319 (1979).

[38] K. Abe et al. (T2K Collaboration), Phys. Rev. D 87, 012001 (2013); 87, 019902(A) (2013).

[39] T. K. Gaisser, G. B. Yodh, V. D. Barger, and F. Halzen, in 14th International Cosmic Ray Conference (ICRC 1975) Munich, Germany, 1975 (Max-Planck Inst. for Extraterrestriche Physik, Munich, 1975), pp. 2161-2166.

[40] J. W. Cronin, R. Cool, and A. Abashian, Phys. Rev. 107, 1121 (1957).

[41] J. V. Allaby et al. (IHEP-CERN Collaboration), Phys. Lett. 30B, 500 (1969).

[42] M. J. Longo and B. J. Moyer, Phys. Rev. 125, 701 (1962).

[43] B. M. Bobchenko et al., Yad. Fiz. 30, 1553 (1979) [Sov. J. Nucl. Phys. 30, 805 (1979)].

[44] V. B. Fedorov, Yu. G. Grishuk, M. V. Kosov, G. A. Leksin, N. A. Pivnyuk, S. V. Shevchenko, V. L. Stolin, A. V. Vlasov, and L. S. Vorobev, Yad. Fiz. 27, 413 (1978) [Sov. J. Nucl. Phys. 27, 222 (1978)].

[45] R. J. Abrams, R. L. Cool, G. Giacomelli, T. F. Kycia, B. A. Leontic, K. K. Li, and D. N. Michael, Phys. Rev. D 1, 1917 (1970).

[46] C. Andreopoulos et al., Nucl. Instrum. Methods Phys. Res., Sect. A 614, 87 (2010); this work uses version 2.12.2, arXiv:0905.2517.

[47] A. S. Meyer, M. Betancourt, R. Gran, and R. J. Hill, Phys. Rev. D 93, 113015 (2016).

[48] J. Nieves, J. E. Amaro, and M. Valverde, Phys. Rev. C 70, 055503 (2004); 72, 019902(E) (2005).

[49] R. Gran, arXiv:1705.02932.

[50] P. Adamson et al. (MINOS Collaboration), Phys. Rev. D 91, 012005 (2015).

[51] A. A. Aguilar-Arevalo et al. (MiniBooNE Collaboration), Phys. Rev. D 83, 052007 (2011).

[52] C. L. McGivern et al. (MINERvA Collaboration), Phys. Rev. D 94, 052005 (2016).

[53] O. Altinok et al. (MINERvA Collaboration), Phys. Rev. D 96, 072003 (2017).

[54] T. Katori, AIP Conf. Proc. 1663, 030001 (2015).

[55] A. Aurisano, C. Backhouse, R. Hatcher, N. Mayer, J. Musser, R. Patterson, R. Schroeter, and A. Sousa (NOvA Collaboration), J. Phys. Conf. Ser. 664, 072002 (2015).

[56] M. Baird, J. Bian, M. Messier, E. Niner, D. Rocco, and K. Sachdev, J. Phys. Conf. Ser. 664, 072035 (2015).

[57] F. Psihas, Measurement of long baseline neutrino oscillations and improvements from deep learning, Ph.D. thesis, Indiana University, 2018.

[58] N. J. Raddatz, Measurement of muon neutrino disappearance with non-fiducial interactions in the NOvA experiment, Ph.D. thesis, Minnesota University, 2016.

[59] A. Aurisano, A. Radovic, D. Rocco, A. Himmel, M. D. Messier, E. Niner, G. Pawloski, F. Psihas, A. Sousa, and P. Vahle, J. Instrum. 11, P09001 (2016).

[60] T. Blackburn, Measurement of $\Delta m_{32}^{2}$ and $\sin ^{2} \theta_{23}$ using muon neutrino and antineutrino beams in the NOvA experiment, Ph.D. thesis, Sussex University, 2019. 
[61] See Supplemental Material at http://link.aps.org/ supplemental/10.1103/PhysRevLett.123.151803 for the muon neutrino distributions in each quartile of hadronic energy fraction, for the profiles of significance surfaces on the $\Delta m_{32}^{2}, \sin ^{2} \theta_{23}, \delta_{C P}$ axes as well as the surfaces computed for the inverted hierarchy.

[62] G. J. Feldman and R. D. Cousins, Phys. Rev. D 57, 3873 (1998).

[63] A. Sousa, N. Buchanan, S. Calvez, P. Ding, D. Doyle, H. Alexander, B. Holzman, J. Kowalkowski, A. Norman, and T. Peterka, in Proceedings of the 23rd International Conference on Computing in High-Energy and
Nuclear Physics (CHEP 2018), Sofia, Bulgaria, 2018 (2019).

[64] K. Abe et al. (Super-Kamiokande Collaboration), Phys. Rev. D 97, 072001 (2018).

[65] M. G. Aartsen et al. (IceCube Collaboration), Phys. Rev. Lett. 120, 071801 (2018).

[66] A. L. Read, J. Phys. G 28, 2693 (2002).

[67] This preference for the upper octant is stronger than the significance of the best fit in the lower octant because binary questions, like the octant and hierarchy, have fewer effective degrees of freedom, analogous to profiling a two-dimensional to one-dimensional confidence interval. 